\title{
Simultaneous surgical operations and hernia repair
}

\author{
V.V. BOYKO ${ }^{1}$, K.Y. PARKHOMENKO ${ }^{2}$, O.E. FESKOV ${ }^{3}$, A.Y. GAVRIKOV ${ }^{3}$, V.I. PIATNOCHKA ${ }^{4}$ \\ 1 "V.T. Zaitsev" Institute of General and Emergency Surgery, \\ National Academy of Medical Sciences of Ukraine, Kharkiv, Ukraine \\ ${ }^{2}$ Kharkiv National Medical University, Kharkiv, Ukraine \\ ${ }^{3}$ Kharkiv Medical Academy of Postgraduate Education, Kharkiv, Ukraine \\ ${ }^{4}$ Department of Surgery of Institute of Postgraduate Education, \\ I. Horbachevsky Ternopil National Medical University, Ternopil, Ukraine
}

\begin{abstract}
The growing spread of combined pathology, especially among the elderly and geriatric population, requires updating of approaches to diagnosis and treatment. In particular, surgical practice has encountered high incidences of combined diseases, requiring operative treatment, in patients with variously localized hernias. In such cases the standard common approach is to treat in stages gradually eliminating certain diseases. But so far, there has been evidence of effective concurrent treatment of combined surgical pathology by conducting simultaneous operations. This review presents the studies of the direct results of simultaneous operations during variously localized hernia plastic repair in combination with other abdominal surgical pathology.
\end{abstract}

Keywords: combined pathology, hernioplasty, simultaneous surgical operations, direct results

\section{INTRODUCTION}

In recent decades, there has been a worldwide increase in the number of patients with combined pathology, which makes it difficult to determine the best tactics of diagnosis and treatment, and creates an additional burden on the health-care system [1]. Combined diseases are particularly challenging in surgical practice. On the one hand, concomitant general somatic diseases increase the risks of adverse events during surgery and the postoperative period, on the other hand, combined surgical pathology requires determining the best treatment tactics [2]. It might be staged treatment with successive surgical interventions or a simultaneous operation with simultaneous elimination of two or more diseases. Despite the reported positive aspects of simultaneous operations, their rate in the structure of surgical interventions is significantly low as compared with the prevalence of combined surgical pathology. Such situations are common in patients with variously localized hernias. So, the studies of feasibility and safety of simultaneous operations during hernioplasty have been still topical.

\section{EPIDEMIOLOGY OF HERNIAS}

Hernioplasty occupies one of the leading places in the structure of elective and emergency abdominal surgery in most countries of the world. The prevalence of hernias is $3-4 \%$ in the general population, but these data have been obtained from the analysis of only individual localizations of hernias in limited samples of patients. It is known that more than 20 million surgical 
interventions for only inguinal hernia are performed in the world annually $[3,4]$. The real prevalence of hernias is difficult to estimate because not all patients seek medical treatment. According to the population study of all the residents of a particular area hernias of anterior abdominal wall in persons older than 10 years has been detected in $20.9 \%$ of the population, including umbilical hernias in $10.2 \%$, inguinal hernias in $8.3 \%$, and incisional hernias in $2.4 \%[5,6]$. According to other data, inguinal hernias $(70.7 \%)$ predominate in operated patients, with much lower prevalence of umbilical and paraumbilical (13.3\%), epigastric $(6.6 \%)$ and other types of hernias [7]. Basing on the results of ultrasound examination, M.A. Bedewi et al. (2012) estimated that the prevalence of umbilical and paraumbilical hernias could reach $25 \%$ [8].

The prevalence of incisional ventral hernias (IVH) can be estimated by the number of abdominal interventions, which reaches 2 million annually, with IVH occurred in $3-11 \%$ of patients. In the United States alone, 350,000 ventral hernias repairs were performed in 2006 [9,10]. According to M.A. Plymale et al. (2017), the cost of every ventral hernia repair is from $\$ 7.5$ to 18.6 thousand, increasing due to the risk factors and the development of postoperative complications [11]. It should be noted that every repeat operation for IVH forms a vicious circle, because the longer the surgery history is, the higher the frequency of repeat operations, the duration of operation, the number of wound complications and recurrences are. During 140 months recurrences were detected in $37 \%$ of patients operated for primary ventral hernia, in $67 \%$ after IVH hernioplasty, in $73 \%$ after IVH repeat hernioplasty [12].

It is even more difficult to estimate the prevalence of internal hernias, in particular, hiatal hernias $(\mathrm{HH})$. In most patients, hernias of this location are asymptomatic, and in many other cases, the manifestations of the disease are not specific and may simulate other diseases [13-16]. But according to the results of some epidemiological studies, it is established that the prevalence of $\mathrm{HH}$ reaches $40 \%$, and $15 \%$ of them require surgical treatment $[17,18]$.

\section{COMBINED SURGICAL PATHOLOGY IN HERNIAS PATIENTS AND HERNIA REPAIR}

The evidence has been obtained so far that in many cases, the formation of hernias, especially of multiple localization and recurrent hernias, is a manifestation of systemic connective tissue dysplasia (DST) [19]. Connective tissue disorder is associated with many other surgical diseases. These include varicose vein disease, biliary dyskinesia with the development of gallstone disease (GSD), gastroesophageal reflux disease (GERD) and $\mathrm{HH}$, gastric and duodenal ulcers, diverticula of di- gestive tract, peritoneal commissures in abdominal cavity and others [20]. L.V. Galimov et al. (2017) identified another abdominal pathology in 211 (68.1\%) out of 310 patients, operated for $\mathrm{HH}$. It was a GSD, benign ovarian tumors, abdominal adhesions and others [21]. The prevalence of combined pathology, requiring operative corrections, is quite high in hernia patients, but surgical tactics in these cases remain discussable.

In recent years, there have been more and more reports of simultaneous operations - surgery under one anesthesia on two or more organs. The advantages of simultaneous operations include the simultaneous elimination of two or more diseases, each of which has a risk of complications; elimination of danger of surgery in the context of adhesions after the previous operation; high economic efficiency; elimination of recurrent preoperative stress due to the expectations of the next operation [22]. Most publications, devoted to simultaneous operations have reported no difference between their immediate results and those of individual operations. However, the number of complications after simultaneous operations is less than their total number after staged interventions [23-25].

One of the most common simultaneous surgical operations in herniology is hernioplasty with abdominoplasty in overweight patients. This approach has not increased the number of complications, but contributed to improving aesthetic and functional results [26]. Simultaneous hernioplasty and antireflux intervention are recommended for patients with obesity and hiatal hernias along with bariatric surgery [27]. H. Garg et al. (2017) reported hernioplasty of $\mathrm{HH}$ to eliminate GERD during bariatric surgery (sleeve gastrectomy) in 5 patients. The symptoms of GERD were eliminated in all patients. So, the authors consider simultaneous hiatal hernioplasty appropriate in obese patients [28].

Simultaneous abdominoplasty during hernioplasty of IVH in patients with abdominal wall defects after bariatric surgery improves the quality of life regarding functional, aesthetic and psychological aspects. The authors believe that such an approach should be a mandatory part of a comprehensive treatment of such patients [29]. A. Moreno-Egea et al. (2016) pointed out that abdominoplasty during hernioplasty of IVH did not increase the duration of inpatient treatment and the frequency of early and late complications, but improved the quality of life as compared with isolated hernioplasty [30]. H.Le Gall et al. (2017) performed simultaneously laparoscopic mesh hernioplasty of umbilical and / or trocar hernia during abdominoplasty, and also did not detect any increase in the incidence of local and other postoperative complications [31]. Abdominoplasty is often combined with operations for gynecological pathology [32].

The good direct results of abdominoplasty with simultaneous repair of ventral hernia with severe ab- 
dominal rectus diastasis were obtained. No complications, except seromas in 2 out of 32 cases, were identified during the postoperative period. The examination, an average of 18 months after surgery, indicated a good cosmetic result, no disease recurrences were detected [33].

However, there have been reports of burdened course of postoperative period and an increase in the incidence of complications $[34,35]$. Thus, it has been estimated that panniculectomy during hernioplasty was associated with wound complications $(O R=1.69$, $P<0.001)$, with unplanned repeat surgery $(O R=2.08$, $P \leq 0.001)$, venous thromboembolism ( $O R=2.48$, $P=0.043)$ and an increase in the incidence of other complications $(\mathrm{OR}=2.08, \mathrm{P}<0.001)$ [36]. P.G. Koolen et al. (2014) estimated the results of simultaneous hernioplasty and abdominoplasty or panniculectomy (36.3\% of 4925 patients) as compared with isolated abdominoplasty or panniculectomy (63.7\% of 4925 patients). According to their data, the incidence of complications in patients with simultaneous hernioplasty was higher (18.3\% vs. 9.8\%). In both groups, the local and general complications were associated with increased BMI. [34]. According to other data, concomitant hernioplasty and panniculectomy in patients with IVH and obesity did not increase the incidence of individual local wound and inflammatory complications, but increased the overall incidence of wound complications as compared with isolated hernioplasty $(46.5 \%$ vs. $27.9 \%$, respectively). The incidence of recurrent hernias did not differ significantly ( $11.6 \%$ vs. $9.3 \%$, respectively). The authors consider hernioplasty and panniculectomy appropriate in patients with apron bellies [37]. The analysis of isolated panniculectomy and panniculectomy along with hernioplasty (143 and 42 patients, respectively) has showed that panniculectomy does not increase the incidence of wound complications (including seromas, skin necrosis, wound revision and debridement, wounds and vacuum aspiration), but increases the incidence of cellulite [38]. It is worth noting that even isolated panniculectomy causes a high risk of wound (hematoma, seroma, wound infection and cellulite) and general complications, including venous thromboembolism [39], for the prevention of which different perioperative measures and technical improvements in surgery are offered [40].

Laparoscopic correction of $\mathrm{HH}$ is often combined with other operations. L.V. Galimov et al. (2017) performed laparoscopic cholecystectomy (LADCHOL) on 154 out of 310 patients, underwent, performed surgery to remove benign ovarian tumors on 29 patients, 25 patients underwent dissection of the ligament of Treitz, 11 patients underwent viscerolysis, and 2 patients underwent repair of hernias of other localizations. Wadhwa et al. (2003) performed 145 simultane- ous operations (combined, according to the authors). Most often they were LADCHOL and ventral hernia repair, combined with operations for gynecological or urological pathology, with fundoplication, etc. The authors did not detect any increase in the incidence of complications and the duration of treatment [41]. However, simultaneous operations for GSD and $\mathrm{HH}$ have not been widely recognized yet, linked to the inadequate preoperative evaluation, insufficient examination of the organs in the abdominal cavity, overestimation of surgical risk and unwillingness of surgeons and anesthesiologists to expand the scope of operation [18].

A. Iljin et al. (2019) analyzed the results of simultaneous hernioplasty and abdominoplasty in the patients who lost weight significantly after bariatric surgery. Impaired wound healing, seromas, pneumonia and dysesthesia of the abdominal wall without a significant difference between onlay and sublay methods were the most common among the postoperative complications [42]. Simultaneous operations are also performed using single-port laparoscopic access [43]. If it is necessary, the laparoscopic access is extended by making an additional incision and with the help of special retractors [44].

V.A. Samartsev et al. (2017) performed simultaneous operations on 14 of 27 patients with large and giant IVH during posterior separation hernioplasty. Those operations included reconstructive colon surgery, transverse abdominoplasty, cholecystectomy and bariatric surgery [45]. Anterior abdominal wall hernias are accompanied very often by pathology of the major arteries in patients of older age. In such cases, it is offered to undergo simultaneous hernioplasty of local tissues without the use of alloplastic materials in order to prevent the risk of infection of a vascular implant. The authors performed such simultaneous interventions on 30 patients, and there were no complications [46].

The possibilities for simultaneous operations have significantly increased due to the active introduction of endovideosurgical methods, providing the possibility of single-access laparoscopic surgery. The most common main pathologies during the simultaneous operations were GSD and umbilical hernias, combined with each other or with adhesive disease, incisional hernia, liver cysts, Meckel's diverticulum, chronic appendicitis. The duration of operation was $80 \pm 10$ minutes, the average bed-day was $3.2 \pm 0.5$, and only one postoperative complication such as postoperative wound infiltrate $(2.5 \%)$ was detected. At the stage of mastering simultaneous operations, the only inconvenience was the "conflict of instruments" [47].

According to the analysis of long-term results of simultaneous laparoscopic, staged laparoscopic and si- 
multaneous traditional surgery for surgical pathology (hernias, chronic and acute cholecystitis) and urological pathology (malignant neoplasm of kidney and prostate), the safety, efficiency and low injury rate of laparoscopic interventions were proved. The severity of postoperative pain was less, physical activity was restored faster, and the quality of life was higher [48]. V.C. Shakya (2015) reported good results of simultaneous LAPCHOL and Morgagni hernia repair through a single laparoscopic approach [49]. LAPCHOL is often performed in combination with operations for gynecological pathology. In addition to good medical results, the socio-economic efficiency of such operations has been established. According to the calculations, the economic benefit was almost $\$ 1,000$. USA per patient [50].

S. Arafat and V. Alsabek reported good results concerning two cases of simultaneous LAPCHOL and transabdominal preperitoneal patch technique (TAPP) of inguinal hernia. The patients were hospitalized due to symptomatic cholecystolithiasis, but the examination revealed a direct inguinal hernia. At the first stage, LAPCHOL was performed, and at the second stage hernioplasty. The authors believe that simultaneous surgery is safe and relieves a patient from a repeat surgery and general anesthesia, but it is appropriate only in the absence of inflammation of the gallbladder [51]. Simultaneous operations during LAPCHOL in 19 patients did not lead to conversion in any case, did not affect the incidence of postoperative complications and the length of hospital stay. The authors consider these operations to be a good alternative to two separate interventions [52]. However, there was a greater impact of simultaneous surgery on the rates of tissue respiration and acid-base composition of blood, as reflected in an increase in carbon dioxide and a decrease in oxygen in the blood. These changes occurred less during the laparoscopic access as compared with the open one [53].

S. Hayakawa et al. (2018) reported the results of simultaneous LAPCHOL and TAPP of inguinal hernia in 17 patients. The average duration of operation was $157 \pm 39$ minutes, the average duration of inpatient treatment was $3.2 \pm 0.6$ days. In two cases the inferior epigastric vessels were damaged during the operation, there was a seroma in one case. There were no other complications. The average cost of treatment was \$ 7673 , while the total cost of individual hernioplasties and LAPCHOL was more than $\$ 10000$. The authors believe that simultaneous surgery is safe and should be standard for combined pathology [54]. Other authors in a series of 22 similar cases reported 1 case of gallbladder perforation, 1 case of ipsilateral testicular atrophy and 1 case of seroma. There were no infectious complications [55].
According to the observations of A. Lehmann et al. (2014) 8 patients underwent simultaneous LAPCHOL and TAPP of inguinal hernias. The average duration of operation was 55 minutes (from 30 to 80 minutes), the average duration of inpatient treatment was 3.6 days. 1-2 trocars were additionally inserted for the performance of LAPCHOL. In one case, a groin hematoma was detected after surgery, and in another case there was postoperative fever. The authors consider simultaneous operation to be a good alternative to two separate operations [56]. In addition, hernioplasty is considered appropriate during urological surgery [57] and colorectal surgery [58]. The authors report that the use of microsurgical techniques has improved the results of simultaneous hernioplasty of inguinal hernias and urological operations (varicocelectomy or denervation of the spermatic cord). According to the observations of the authors, hematoma occurred in $0.85 \%$ of cases, there were no other complications and hernia recurrences [57].

The increase in the duration of surgery is mentioned very often as one of the negative consequences of simultaneous operations. Thus, hernioplasty of ventral hernias with the use of a mesh during colorectal interventions increased the duration of surgery up to 195.8 \pm 98.7 as compared with $164.3 \pm 84.4$ min during isolated colorectal interventions. However, this did not affect postoperative mortality, the incidence of complications and the duration of inpatient treatment. Thus, it indicates the feasibility of such interventions [56]. The results of other studies also show that simultaneous operations lead to an increase in the duration of surgery and anesthesia, as well as to an increase in the incidence of adverse events and the duration of hospitalization. In particular, it was observed in case of simultaneous LAPCHOL and laparoscopic bariatric surgery [59]. W. Ueland et al. (2020) found that additional surgical procedures were also a risk factor for an increase in the duration of hospitalization [60].

The reason for refusing to perform simultaneous operations is also explained by the increase in logical risks of anesthesia due to the increase in the duration of operation, the increase in the number of critically ill patients, and the inadequate evaluation of a patient before the operation. In the context of laparoscopic surgery, prolonged exposure to intense carboxypneumoperitoneum and anesthesia increases the anesthetic risk, and in the context of open operations and the need for additional access, the surgical injury increases [22]. E.M. Altmark believes that the main reasons for a small number of simultaneous operations are insufficient examination, insufficient intraoperative revision, the tendency of surgeons to perform multi-staged interventions, the unwillingness of surgeons and anesthesiologists to perform advanced operations [61]. At 
the same time, modern possibilities of anesthesia, multimodal anesthesia, thorough preoperative preparation allow to reduce risks during even long and extensive surgery [22].

\section{CONCLUSIONS}

To sum up, patients with hernias have a fairly high incidence of other surgical diseases, which also require surgical treatment. In cases of such combined patholo- gy a surgeon faces a problem of choice of treatment tactics, such as a simultaneous surgical operation or consecutive operations during individual hospitalizations. The studies devoted to the effectiveness of a particular tactic are not numerous and are based on the analysis of a small set of observations, and their results are very contradictory. Therefore, further studies of the feasibility and effectiveness of simultaneous operations are topical.

Conflict of interest: none declared

Financial support: none declared

\section{REFERENCES}

1. Kryvokulsky BD, Zhulkevich IV, Kryvokulsky DB, Shkrobot LV. Studying the influence of age and comorbidity index on the thrombotic complications risk in patients with endometrial cancer at preoperative stage. Bull Sci Res. 2018;2:150-4.

2. Zhulkevych IV, Kryvokulsky BD. Personalization in oncology: individual approach to the prevention of thromboembolic complications during hysterectom. Bull Soc Hygiene and Health Protection Organization of Ukraine. 2019;4:11-8.

3. Hernia Surge Group. International guidelines for groin hernia management. Hernia. 2018;22(1):1-165.

4. Nimptsch U, Mansky T. Deaths following cholecystectomy and herniotomy - an analysis of nationwide German hospital discharge data from 2009 to 2013. Dtsch Arztebl Int. 2015;112:535-43.

5. Kirienko AI, Shevtsov YN, Nikishkov AS, et al. Prevalence of hernias of the anterior abdominal wall: results of a population study. Surgery. NI Pirogov J. 2016;8:61-6.

6. Sazhin A, Zolotukhin I, Kirienko A, et al. Prevalence and risk factors for abdominal wall hernia in the general Russian population. Hernia. 2019;23(3):1-6

7. Dabbas N, Adams K, Pearson K, Royle GT. Frequency of abdominal wall hernias: is classical teaching out of date? JRSM Short Rep. 2011;2(1):5.

8. Bedewi MA, El-Sharkawy MS, Al Boukai AA, Al-Nakshabandi N. Prevalence of adult paraumbilical hernia. Assessment by high-resolution sonography: a hospitalbased study. Hernia. 2012;16:59-62.

9. Shelton J, Poulose BK, Phillips S, et al. Epidemiology and cost of ventral hernia repair: making the case for hernia research. Hernia. 2012;16:179-83.

10. Poulose BK, Shelton J, Phillips S. et al. Epidemiology and cost of ventral hernia repair: making the case for hernia research. Hernia. 2012;16:179-83.

11. Plymale MA, Ragulojan R, Davenport DL, Roth JS. Ventral and incisional hernia: the cost of comorbidities and complications. Surg Endosc. 2017;31(1):341-51.
12. Holihan JL, Alawadi Z, Martindale RG, et al. Adverse events after ventral hernia repair: the vicious cycle of complications. J Am Coll Surg. 2015;221(2):478-85.

13. Lebenthal A, Waterford SD, Fisichella PM. Treatment and controversies in paraesophageal hernia repair. Front Surg. 2015;2:13

14. Carrott PW, Hong J, Kuppusamy M, Koehler $\mathrm{RP}$, Low DE. Clinical ramifications of giant paraesophageal hernias are underappreciated: making the case for routine surgical repair. Ann Thorac Surg. 2012;94(2):421-8.

15. Carrott PW, Markar SR, Hong J, et al. Iron-deficiency anemia is a common presenting issue with giant paraesophageal hernia and resolves following repair. J Gastrointest Surg. 2013;17(5):858-62.

16. Dellaportas D, Papaconstantinou I, Nastos C et al. Large paraesophageal hiatus hernia: is surgery mandatory? Chirurgia (Bucur). 2018;113(6):765-71.

17. Zyabreva IA, Dzhulay TE. Hernia of the esophageal opening of the diaphragm: controversial, unresolved and promising aspects of the problem (literature review). Upper Volga Med J. 2015;14(4):24-8.

18. Chernousov A, Khorobrikh T, Vetshev F, Melentyev A. Surgical treatment of cholelithiasis, combined with hernia of the esophageal opening of the diaphragm. Doctor. 2012;10:2-7.

19. Henriksen NA. Systemic and local collagen turnover in hernia patients. Dan Med J. 2016;63(7):B5265.

20. Systemic pathology of connective tissue: a guide for doctors. In: Stroyev Yul, Churilova LP ed. SPb: ELBISPb. 2014:368 p.

21. Galimov LV, Khanov VO, Mamadaliev DZ, et al. Creative surgery of hiatal hernia. Surgery. 2017;7:30-2.

22. Semyonov VV, Kurygin AA. Simultaneous operations on the abdominal organs: controversial and obvious aspects of the problem. Bull Surg. 2014;173(6):96-99.

23. Murodov Al, Kadyrov FOR. Simultaneous video endoscopic operations for combined diseases of the abdominal and retroperitoneal organs. Med Bull Bashkortostan. 2017;12(3(69)):129-34.

24. Herbali OY. Simultaneous operations in patients with chronic calculous cholecystitis. Endoscopic Surg. 2014;6:26-8.

25. Orr NT, Davenport DL, Roth JS. Outcomes of simultaneous laparoscopic cholecystectomy and ventral hernia repair compared to that of laparoscopic cholecystectomy alone. Surg. Endosc. 2013;27(1):67-73

26. Dessy LA, Mazzocchi M, Fallico N, et al. Association between abdominal separation and inguinal or crural hernias: our experience and surgical indications. J Plast Surg Hand Surg. 2013;47(3):209-12.

27. Soricelli E, Casella G, Rizzello M et al. Initial experience with laparoscopic crural closure in the management of hiatal hernia in obese patients undergoing sleeve gastrectomy. Obes Surg. 2010;20:1149-53.

28. Garg H, Vigneshwaran B, Aggarwal S, Ahuja V. Impact of concomitant laparoscopic sleeve gastrectomy and hiatal hernia repair on gastro-oesophageal reflux disease in morbidly obese patients. J Minim Access Surg. 2017;13(2):103-8.

29. Iljin A, Antoszewski B, Durczyński A, et al. Long-term results of incisional hernia repair with concomitant abdominoplasty in postbariatric patients. Pol Przegl Chir. 2016;88:147-54.

30. Moreno-Egea A, Campillo-Soto Á, Morales-Cuenca $\mathrm{G}$. does abdomino $\urcorner$ plasty add morbidity to incisional hernia repair? A randomized controlled trial. Surg Innov. 2016;23(5):474-80.

31. Le Gall H, Reibel N, De Runz A, et al. Abdominoplasty and simultaneous laparoscopic ventral hernia repair. Clinical study about 45 patients. Ann Chir Plast Esthet. 2017;62(2):115-21.

32. Filho JMT, Maciel S, Belerique M. Abdominoplasty combined with intraabdominal gynecologic surgery. Rev. Soe. Bras. CiroPlást. 2004;19(2):41-52.

33. Cheesborough JE, Dumanian GA. Simultaneous prosthetic mesh abdominal wall reconstruction with abdominoplasty for ventral hernia and severe rectus diastasis 
repairs. Plast Reconstr Surg. 2015; 135:268-76.

34. Koolen PG, Ibrahim AM, Kim K, et al. Patient selection optimization following combined abdominal procedures: analysis of 4925 patients undergoing panniculectomy/ abdominoplasty with or without concurrent hernia repair. Plast Reconstr Surg. 2014;134(4):539e-50e.

35. Ortega J, Navarro V, Cassinello N, Lledo S. Requirement and postoperative outcomes of abdominal panniculectomy alone or in combination with other procedures in a bariatric surgery unit. Am J Surg. 2010;200:235-40.

36. Fischer JP, Tuggle CT, Wes AM, Kovach SJ. Concurrent panniculectomy with open ventral hernia repair has added risk versus ventral hernia repair: an analysis of the ACS-NSQIP database. J Plast Reconstr Aesthet Surg. 2014;67(5):693-701.

37. Warren JA, Epps M, Debrux C, et al. Surgical site occurrences of simultaneous panniculectomy and incisional hernia repair. Am Surg. 2015;81(8):764-9.

38. Zemlyak AY, Colavita PD, El Djouzi, S et al. Comparative study of wound complications: isolated panniculectomy versus panniculectomy combined with ventral hernia repair. J Surg Res. 2012; 177(2):387-91.

39. Winocour J, Gupta V, Ramirez JR, et al. Abdominoplasty: risk factors, complication rates, and safety of combined procedures. Plast Reconstr Surg. 2015;136:597e-606e.

40. Dutot MC, Serror K, Al Ameri O, et al. Improving safety after abdominoplasty: a retrospective review of 1128 cases. Plast Reconstr Surg. 2018;142:355-62.

41. Wadhwa A, Chowbey PK, Sharma A, et al. Combined procedures in laparoscopic surgery. Surg Laparosc Endosc Percutan Tech. 2003;13(6):382-6.

42. Iljin A, Antoszewski B, Zieliński T, et al. Sublay or onlay incisional hernia repair along with abdominoplasty: which is better? Long-term results. Hernia. 2019;23:757-65.
43. Kim G, Lomanto D, Lawenko MM, et al. Single-port endo-laparoscopic surgery in combined abdominal procedures. Asian J End Surg. 2013;6(3):209-13.

44. Umeda S, Hachisuka T, Otsu T, et al. Reduced-port endo-laparoscopic surgery using umbilical zigzag incision for concomitant operations: A case serie. Int J Surg Case Reports. 2018;51:170-3.

45. Samartsev VA, Gavrilov VA, Parshakov AA, Kuznetsova MV. Posterior separation hernioplasty TAR for incisional ventral hernias W3. Perm Medical Journal. 2017;34(1):35-42.

46. Miroshnichenko PV, Bolshak OA, Stroilo AB, et al. Simultaneous operations in the surgical treatment of diseases of the main vessels. Transport Medicine of Ukraine. 2008; 1:29-32.

47. Rakhmatullaev RR, Rakhmatullaev AR, Khasanov SM, Ibragimov SB. Simultaneous operations from a single laparoscopic access. Bull Acad Med Sci Tajikistan. 2017;1:77-7.

48. Murodov AI, Kadyrov ZA, Odilov AYU, Aliyev ZO. Quality of life of patients with combined diseases of the abdominal cavity and retroperitoneal space after simultaneous video endoscopic and traditional operations. Med Bull Bashkortostan. 2017;12(3) (69):48-51.

49. Shakya VC. Simultaneous laparoscopic management of Morgagni hernia and cholelithiasis: two case reports. BMC Res Notes. 2015;8:283.

50. Aboyan IA, Aboyan ME, Borodin VG, et al. Socio-economic efficiency of simulated operations in women of reproductive age. Pract Med. 2013;7(76):92-6.

51. Arafat S, Alsabek B. Simultaneous laparoscopic cholecystectomy and transabdominal preperitoneal hernioplasty: two case reports evaluate the safety and surgical complications. Clin Case Rep. 2017;5(12):2093-6.

52. Caglià $\mathrm{P}$, Tracia A, Amodeo L, et al. Laparoscopic cholecystectomy and concomitant diseases effectiveness of the single step treatment. Ann Ital Chir. 2015;86:524-7.

53. Malik JV, Al Shirafi MA. Features of tissue respiration parameters in simultaneous surgery in patients with gallstone disease. Probl Ecol Med. 2010;14(5-6):25-8.

54. Hayakawa S, Hayakawa T, Inukai K, et al. Simultaneous transabdominal preperitoneal hernia repair and laparoscopic cholecystectomy: A report of 17 cases. Asian J Endosc Surg. 2018;8.

55. Quezada N, Maturana G, Pimentel E et al. Simultaneous TAPP inguinal repair and laparoscopic cholecystectomy: results of a case series. Hernia. 2019;23(1):119-23.

56. Lehmann A, Piątkowski J, Nowak M, et al. Simultaneous TAPP (transabdominal pre-peritoneal technique) for inguinal hernia and cholecystectomy - a feasible and safe procedure. Pol Przegl Chir. 2014;86(2):73-6.

57. Schulster ML Cohn MR, Najari BB, Goldstein M. Microsurgically assisted inguinal hernia repair and simultaneous male fertility procedures: rationale, technique and outcomes. J Urol. 2017;198(5):1168-74.

58. Benlice C, Gorgun E, Aytac E, et al. Mesh herniorrhaphy with simultaneous colorectal surgery: a case-matched study from the American College of Surgeons National Surgical Quality Improvement Program. Am J Surg. 2015;210(4):766-71.

59. Dorman RB, Zhong W, Abraham AA, et al. Does concomitant cholecystectomy at time of Roux-en-Y gastric bypass impact adverse operative outcomes? Obes Surg. 2013;23(11):1718-26.

60. Ueland W, Walsh-Blackmore S, Nisiewicz M, et al. The contribution of specific enhanced recovery after surgery (ERAS) protocol elements to reduced length of hospital stay after ventral hernia repair. Surg Endosc. 2020;34(10):4638-44.

61. Altmark EM. Simultaneous laparoscopic operations. Bull Surg. 2007;166(4):111-3. 